\title{
Transgelin is a marker of repopulating mesangial cells after injury and promotes their proliferation and migration
}

\author{
Christoph Daniel ${ }^{1}$, Andrea Lüdke ${ }^{2}$, Andrea Wagner ${ }^{3}$, Vladimir T Todorov ${ }^{3}$, Bernd Hohenstein ${ }^{3}$ and Christian Hugo ${ }^{3}$
}

\begin{abstract}
Mesangial cell (MC) migration is essential during glomerular repair and kidney development. The aim of the study was to identify marker/player for glomerular progenitor/reserve cells migrating into the glomerulus after MC injury and during glomerulogenesis in the rat. Experimental mesangial proliferative nephritis was induced in Sprague Dawley rats by intravenous injection of OX-7 antibody. We investigated mRNA expression profiles in isolated glomeruli from on days 0,1 , 2, 3, and 5 after induction of anti-Thy 1 nephritis using Affymetrix microarray technology. Using self-organizing maps, transgelin was identified as a new marker for repopulating glomerular cells. Expression of transgelin during anti-Thy 1 nephritis was investigated by northern blot, real-time PCR, western blot, and immunohistochemistry. Migration and proliferation assays using isolated MCs after transgelin knockdown by siRNA were performed to investigate the potential role of transgelin during glomerular repopulation. Transgelin mRNA was not detected in healthy glomeruli. It was strongly upregulated during the repopulation process starting on day 1, continued to be increased until day 5 and disappeared on day 7. Transgelin was specifically expressed at the edge of the migratory front during glomerular repopulation as indicated by transgelin/OX-7 double staining. Transgelin expression was similar in migrating vs non-migrating MCs in vitro. Blocking of transgelin expression by siRNA treatment resulted in inhibition of MC migration and proliferation. Transgelin was also expressed in MCs during glomerulogenesis and in biopsies from patients with IgA nephritis. In conclusion, transgelin in the kidney is upregulated in repopulating MCs in vivo and supports their migratory and proliferative repair response after injury.

Laboratory Investigation (2012) 92, 812-826; doi:10.1038/labinvest.2012.63; published online 2 April 2012
\end{abstract}

KEYWORDS: anti-Thy1 glomerulonephritis; glomerulus; kidney; repair mechanism; gene expression profiling

Glomerulonephritis remains a very common cause of endstage renal disease. Many pathophysiological events such as phenotype changes, inflammation, mesangial cell (MC) injury and proliferation, and matrix expansion of the most frequent mesangial proliferative glomerulonephritis in man, IgA nephropathy, are mimicked by the mesangial proliferative glomerulonephritis model in the rat (anti-Thy1 model). In addition, the reversible anti-Thyl model is a welldefined model to study the conditions and time course of a successful glomerular healing reaction after severe injury within a few days. ${ }^{1,2}$ In contrast, a standardized examination of human IgA nephropathy will always remain impossible due to the lack of a known starting point of disease, the marked variability of the disease course, entity and ethical problems associated with repetitive biopsies without direct therapeutical consequences.

With the rapid identification of 30000-40 000 genes in the Human Genome Project a catalogue of possible therapeutic targets for medicine has been set up, but the function of these genes and their relationship to pathways in health and disease needs to be identified. Herein, microarray technology has become a standard tool for generation of gene expression profiles in various types of tissue. ${ }^{3}$ Gene expression profiles have been generated in experimental ${ }^{4}$ or human renal diseases such as lupus nephritis ${ }^{5}$ or focal segmental glomerulosclerosis, ${ }^{6}$ in circulating leukocytes of patients with different renal diseases, ${ }^{7}$ in streptozotocin-induced diabetic nephropathy, ${ }^{8}$ and anti-glomerular basement membrane

\footnotetext{
${ }^{1}$ Division of Nephropathology, University of Erlangen-Nürnberg, Erlangen, Germany; ${ }^{2}$ Division of Nephrology and Hypertension, University of Erlangen-Nürnberg, Erlangen, Germany and ${ }^{3}$ Division of Nephrology, University of Dresden, Dresden, Germany

Correspondence: Dr C Daniel, PhD, Division of Nephropathology, University of Erlangen-Nürnberg, Krankenhausstrasse 8-10, 91054 Erlangen, Germany. E-mail: christoph.daniel@uk-erlangen.de 
GN. ${ }^{9}$ Izumi et al ${ }^{10}$ investigated the gene expression profile on certain days of experimental mesangial proliferative GN.

Using microarray technology, we tried to link alterations in glomerular gene expression profiles to potential functional importance focusing on the early repair reaction after MC injury. During this phase, severe complement-dependent mesangiolysis (day 1) with maximal influx of inflammatory cells is followed by the coordinated migration and proliferation of glomerular cells (days 2-5) from the hilus towards the glomerular periphery, which is accompanied by a substantial mesangial matrix expansion. ${ }^{1,2}$ While studies using bone marrow transplantation suggested a minor participation of bone marrow-derived cells in this process in the anti-Thyl model, about $90 \%$ of the repopulating cells are locally derived. ${ }^{11}$ While this early and complex glomerular repopulation process during the first week is critical for the later outcome of disease, ${ }^{12}$ the later time course is dominated by a resolution of excess cells and matrix via apoptosis and matrilysis, respectively. In this study, we were especially interested in the further characterization of these migrating glomerular cells after injury. Hereby, the injury phase (day 1) as well as the starting (days 2 and 3 ) and pronounced (day 5) repair phase was compared with normal healthy glomeruli. Using SOM (self-organizing map) clustering techniques to obtain genes characteristically regulated in certain patterns during these different phases, we were able to identify genes likely being involved in distinct processes during injury and repair. Since we focused on the mechanism of glomerular repopulation, transgelin a novel marker for repopulating MCs was identified within the expected SOM cluster and further characterized.

\section{MATERIALS AND METHODS}

\section{Experimental Mesangial Proliferative} Glomerulonephritis and Kidney Development

Male Sprague Dawley rats weighing 180-200 g were purchased from Charles River, Sulzfeld, Germany and housed under specific pathogen-free conditions in our own animal facilities. The animal studies were performed in accordance with the internal animal review board (Regierung von Mittelfranken: 621-2531.31-19/98).

Experimental mesangial proliferative GN (anti-Thyl model) was induced by a single i.v. tail injection of $1 \mathrm{mg} / \mathrm{kg}$ of body weight of mouse monoclonal anti-Thy1 antibody OX-7 (European Collection of Animal Cell Culture, Salisbury, UK). The time course of the GN model has been described in detail in Introduction. Nephritic rats were killed on days 1, 2, 3, and 5 after anti-Thyl antibody injection $(n=2-3$ animals per time point). Untreated age- and weight-matched rats were used as day 0 controls. For verification of the microarray data, anti-Thyl nephritis was induced in a second experiment. Nephritic rats were killed on days 3, 5, and 7 for preparation of glomerular RNA and glomerular proteins ( $n=5$ per time point).
Expression of transgelin was also investigated during renal development using kidneys from six newborn rats collected 6 days after birth. In contrast to glomerulogenesis in man, renal development in the rat is still ongoing during the first 2 weeks after birth. In these biopsies, s-shape bodies can be found in the outer cortex and nearly full-developed glomeruli in the inner cortex.

\section{Source of Human Renal Tissue}

Archival tissues from core needle biopsies performed between 2006 and 2010 at the Medical Clinic 4 (Erlangen, Germany) were used for this study. The morphological diagnosis of IgA nephropathy was made by the local pathologist who unaware of study findings. For this study, we used eight biopsies showing early and additional eight biopsies with progressed IgA nephropathy. The mean age of the patients (about 54 years) and the genders (female/male: $3 / 5$ in early $\operatorname{IgA}$ nephropathy and 2/6 in late IgA nephropathy) were comparable in both groups.

Control tissues without evidence of renal disease $(n=10)$ were obtained from distant portions of kidneys surgically excised because of the presence of a localized neoplasm.

\section{Preparation of Glomerular Total RNA and Gene Expression Analysis by Microarray}

Immediately after kill, glomeruli were isolated from rat kidneys by standard sieving method as previously described. ${ }^{13}$ Total RNA was extracted using the RNeasy RNA isolation system (Qiagen, Hilden, Germany) according to manufacturer's instructions and quantified by spectrophotometry (Ultrospec 3000 pro, Amersham Biosciences, Freiburg, Germany). All RNA samples showed A260/280 ratios between 1.9 and 2.1. RNA integrity was determined using an Agilent 2100 Bioanalyzer (Agilent Technologies, Böblingen, Germany) and only high quality RNA $(28 \mathrm{~S} / 18 \mathrm{~S}>1.8)$ was used for further analysis. Target generation for microanalysis was done in cooperation with L Klein-Hitpass (Institute of Cell Biology, University of Essen, Germany).

Briefly, $9 \mu \mathrm{l}(13.5 \mu \mathrm{g})$ of total RNA was used as template in the first-strand cDNA synthesis reaction. The synthesis of double-stranded cDNA and the generation of the biotinylated cRNA was done according to Durig et al. ${ }^{14}$ Fragmentation of cRNA, hybridization of samples to Rat Expression Set 230A Affymetrix GeneChip microarrays (Affymetrix, Santa Clara, CA), washing, staining and scanning of the arrays in a GeneArray scanner (Agilent, Palo Alto, CA) were performed as recommended in the Affymetrix Gene Expression Analysis Technical Manual, 2000 (http://www. affymetrix.com/support/index.affx).

\section{Gene Expression Profile Analysis}

Raw data were analysed using Affymetrix Microarray Suite version 5.1. (MAS v.5.1). The signal intensity represents a measure of the frequency of a transcript in the analysed sample. The detection $P$-value of a transcript determines the 
detection call, which indicates whether a transcript is reliably detected (present) or not detected (absent). Detection of $P$-values between 0 and 0.04 and between 0.06 and 1 results in an absent call (A) and in a present call (P), respectively. For reliable comparisons of multiple arrays, the data were normalized by 'global scaling'.

Differences in gene expression between control and experimental samples were analysed using Affymetrix Data mining tool (DMT) software. For each probe set, a signal log ratio (SLR) is determined as well as a change $P$-value that describes whether there is a change call increase (I), marginal increase (MI), no change (NC), marginal decrease (MD), or decrease (D). For the SLR values, the signal intensities were converted by taking the logarithm of base two. P-values were determined by one-sided Wilcoxon's signed rank test. $R$-squared values $\left(R^{2}\right)$ determined by Pearson Product Moment Correlation measure the strength and direction of linear relationship between the $X$ and $Y$ variables.

\section{Northern-Blot Analysis of Transgelin Expression}

Total RNA was extracted from glomerular preparations of rat kidneys on days $0,3,5$, and 7 after induction of experimental mesangial proliferative GN. Isolation of glomeruli and northern-blot analysis using $20 \mu \mathrm{g}$ total RNA per lane were done as previously described. ${ }^{13}$ The blots were hybridized with an 1.0-kb rat transgelin (SM actin 22-alpha) cDNA fragment kindly provided by Dr C Shanahan (Cambridge, UK). ${ }^{15}$

\section{Western-Blot Analysis}

The proteins were extracted from isolated glomeruli or isolated MCs using $50 \mathrm{mM}$ Tris, $1 \%(\mathrm{v} / \mathrm{v})$ Nonidet P-40, 0.25 $(\mathrm{w} / \mathrm{v})$ sodium deoxycholate, $150 \mathrm{mM} \mathrm{NaCl}, 1 \mathrm{mM}$ EGTA, $1 \mathrm{mM} \mathrm{Na}_{3} \mathrm{VO}_{4}, 1 \mathrm{mM} \mathrm{NaF}$, and proteinase inhibitor cocktail (Complete; Boehringer, Mannheim, Germany) as extraction buffer. For a better solubility, extracts were sonified for $30 \mathrm{~s}$ at $50 \%$ power and $50 \%$ duty cycle using a Sonoplus HD70 (Bandelin, Berlin, Germany). Twenty micrograms of protein were resolved in sodium dodecyl sulphate-polyacrylamide gel electrophoresis using gels containing 12\% acrylamide for analysing transgelin (Abcam, Cambridge, UK). Proteins were transferred semidry onto a nitrocellulose membrane within $1 \mathrm{~h}$ at $1.5 \mathrm{~mA} / \mathrm{cm}^{2}$. Beta-actin (Abcam) was detected simultaneously as loading control. Secondary peroxidase conjugated antibodies were all purchased from Amersham Biosciences (Buckinghamshire, UK).

\section{Renal Morphology and Immunohistochemistry}

Renal biopsies were fixed in methyl Carnoy's solution, embedded in paraffin, cut into $3 \mu \mathrm{m}$ tissue sections and stained as described elsewhere. ${ }^{16}$ Detection was done either by immunofluorescence or by indirect immunoperoxidase staining. Negative controls for immunostaining were performed by deleting the primary antibody.
The following primary antibodies were used for immunohistochemistry: mouse monoclonal anti-Thyl antibody OX-7 (Serotec, Düsseldorf, Germany); two different antibodies against transgelin were used, a goat polyclonal antitransgelin (Ab 10135) and a rabbit anti-transgelin (Ab 14106, both from Abcam). Specificity of both antibodies was confirmed by western-blot analysis (see Supplemental Material).

Alexa Fluor 488 donkey anti-goat IgG $(\mathrm{H}+\mathrm{L})$, Alexa Fluor 555 donkey anti-mouse IgG $(\mathrm{H}+\mathrm{L})$ (Invitrogen, Karlsruhe, Germany) and biotinylated horse anti-mouse $\operatorname{IgG}(\mathrm{H}+\mathrm{L})$ (Vector Laboratories, Linaris, Wertheim-Bettingen) were used as secondary antibodies.

\section{Cell Culture}

Mouse MCs were cultured from isolated glomeruli using standard sieving procedure ${ }^{17}$ and characterized by positive staining for $\alpha$-SM actin and $\alpha 8$-integrin and the lack of the podocyte marker synaptopodin, endothelial marker CD31, and the macrophage/monocyte marker F4/80. MC was grown in DMEM containing $10 \% \mathrm{FCS}$, insulin $(5 \mu \mathrm{g} / \mathrm{ml})$, and penicillin/streptomycin at $37^{\circ} \mathrm{C}$ and $5 \% \mathrm{CO}_{2}$.

\section{Flow Cytometry Analysis}

Transgelin expression was analysed by flow cytometry analysis using the goat anti-transgelin antibody (Abcam). For investigation of transgelins role during MC proliferation, we simultaneously stained for DNA using propidium iodide and transgelin. Transgelin expression was investigated in growing, non-synchronized MC. MC was fixed overnight in ice-cold $65 \%$ methanol after detachment of growing cells from petri dishes by trypsin treatment. After washing with PAB buffer (PBS containing $2 \%$ BSA and $0.01 \%$ sodium azide), cells were permeabilized with $0.1 \%$ Triton X-100 in PBS for $15 \mathrm{~min}$ at $37^{\circ} \mathrm{C}$ followed by washing with $\mathrm{PAB}$ buffer. For staining of DNA, RNA was digested by RNAse A $(1 \mathrm{mg} / \mathrm{ml}$ in PBS) for $12 \mathrm{~min}$ at $37^{\circ} \mathrm{C}$ and $40 \mathrm{~min}$ at room temperature. After washing with $\mathrm{PAB}$ buffer, cells were incubated with anti-transgelin antibody (diluted 1:500 in PAB buffer for $2 \mathrm{~h}$ at $4{ }^{\circ} \mathrm{C}$ ), washed again with $\mathrm{PAB}$ buffer followed by incubation with an Alexa Fluor 488 donkey anti-goat $\operatorname{IgG}(\mathrm{H}+\mathrm{L})$ antibody (Invitrogen, Karlsruhe, Germany). After removal of non-bound antibody, MCs were stained with propidium iodide $(50 \mu \mathrm{g} / \mathrm{ml}$ in PBS) and analysed by flow cytometry analysis (EPICX XL; Beckman-Coulter, Krefeld, Germany) using EXPO32 ADC software (Beckman-Coulter). Cells were defined as transgelin negative when a similar FACS signal was detected than in controls without primary antibody. DNA staining by propidium iodide was used for cell-cycle analysis. Percentages of G1, S, and G2 cell-cycle phases were analysed using Multicycle software (Phoenix Flow Systems, San Diego, USA).

\section{Transfection with Transgelin siRNA}

Mouse MC was transfected with two different siRNAs against transgelin, a negative control siRNA or transfection reagent 
alone all purchased by Invitrogen (Philomath, OR, USA). Cells were transfected with 1 and $10 \mathrm{nM}$ siRNA each using Hiperfect (Qiagen) as transfection reagent following manufacturer's instructions. All tested transgelin siRNA concentrations were able to downregulate transgelin $72 \mathrm{~h}$ after transfection. Best specific effects without off-target effects were achieved using $10 \mathrm{nM}$ siRNA. Consequently, experiments using $10 \mathrm{nM}$ siRNA were shown. Downregulation of transgelin was evaluated by western-blot analysis as previously described ${ }^{18}$ and cells were tested in proliferation and migration assays.

\section{Proliferation Assay}

Proliferation of MCs treated either with transgelin siRNA or with control siRNA was monitored by Bromodeoxyuridine (BrdU) uptake during $\mathrm{S}$ phase using a cell proliferation ELISA (colorimetric) purchased from Roche Diagnostics $\mathrm{GmbH}$, Mannheim, Germany following manufacturer's instructions. Two-thousand siRNA transfected mouse MCs were seeded per well of a microtiter plate and starved for 2 days in culture medium supplemented with $0.1 \%$ FCS. Pulsing with BrdU was done for $4 \mathrm{~h} 1$ day after stimulation with $10 \%$ FCS. Proliferative cell activity of a representative experiment was shown. All assays were done in sextuplicate and each experiment was done a minimum of three times.

\section{In-Vitro Cell Migration Assay}

MC migration was investigated using a Transwell 48-well modified Boyden chamber and $0.1 \%$ gelatine-coated polyvinyl-pyrrolidone-free polycarbonate membranes with $8 \mu \mathrm{m}$ pore size (Neuroprobes, Gaithersburg, MD, USA). Two days after transfection with control siRNA or transgelin-specific siRNA 28000 mouse MC per well were loaded into the upper chamber. The bottom wells were filled with $40 \mu \mathrm{g} / \mathrm{ml}$ fibronectin, used as the chemoattractant, or $0.1 \%$ BSA, used as a negative control. The Boyden chamber was incubated for $5 \mathrm{~h}$ at $37^{\circ} \mathrm{C}$ and $5 \% \mathrm{CO}_{2}$ to allow migration of cells through the membrane. Cells that had transmigrated were stained with Qick diff and three fields of vision per well were analysed using phase contrast microscopy at a $\times 250$ magnification. All assays were done in triplicate and each experiment was done a minimum of three times.

\section{RESULTS}

\section{Identification of Individual Gene Expression Clusters by SOMs during Experimental Mesangial Proliferative Glomerulonephritis}

The anti-Thyl model is a well-characterized rat model of mesangial proliferative nephritis as described in Introduction. ${ }^{19}$ Its well-defined histological disease course ${ }^{1,2}$ is ideally suited to identify genes with a specific expression pattern most likely related to a specific biological process. Repopulation of the glomerulus via proliferating and migrating mesangial reserve cells ${ }^{1,2}$ is an important feature of this model system starting after day 1 and ceasing after day 5 , when the glomerular architecture is markedly restored.

There are several supervised and unsupervised methodologies available to analyse RNA expression data sets. Instead of defining typical gene expression profiles upfront to identify marker genes of certain biological processes, we preferred an unsupervised neural network learning algorithm, the socalled SOMs ${ }^{20,21}$ to characterize the components of our data set and find internal (instead of artificial external) structures and relationships. The Affymetrix DMT software contains a standard implementation of the SOM algorithm to cluster genes or samples.

In the above-mentioned data set (12540 probe sets, ie, $70 \%$ of the probe sets represented on the Rat Expression Set 230A Affymetrix GeneChip array) six different clusters of genes each sharing similar gene expression profiles are identified by SOM algorithm. In Figure 1, the gene expression profiles characteristic for each cluster is depicted. Two clusters seem to be representative for the three major biological processes regarding glomerular repair.

First, clusters 3 and 6 (Figure 2; Table 1) with predominant early changes of gene expression represent the disease switch from injury to repair. Second, clusters 2 and 4 (Figure 2; Table 1) most likely relate to genes characteristic for the

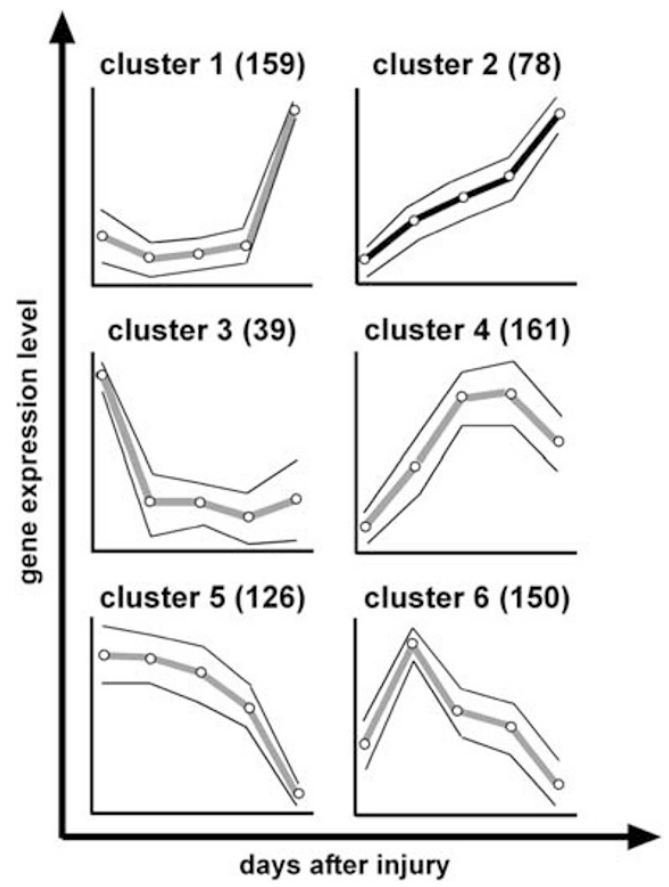

Figure 1 Unsupervised clustering of probe sets using self-organizing maps (SOM) in anti-Thy 1 nephritis. In all, 12540 probe sets are clustered according to their gene expression profile into six different clusters, whereby each cluster represents an individual gene expression pattern over the experimental period. The data points correspond to days $0,1,2,3$, and 5 after injury indicated on the $x$ coordinate. The middle and the two outer graph lines represent the average gene expression pattern and the standard deviation of expression for the cluster, respectively. The number of cluster members is given in parenthesis. 

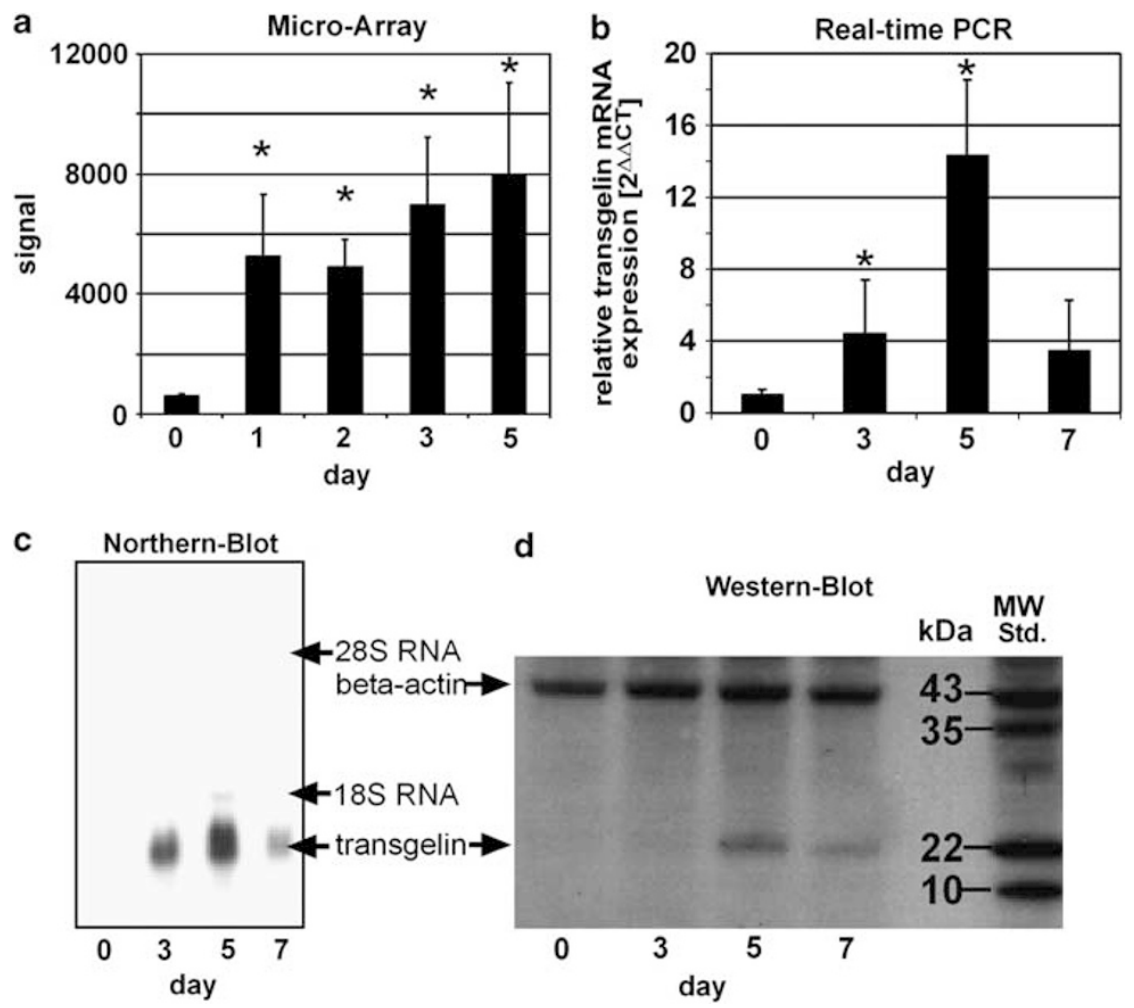

d

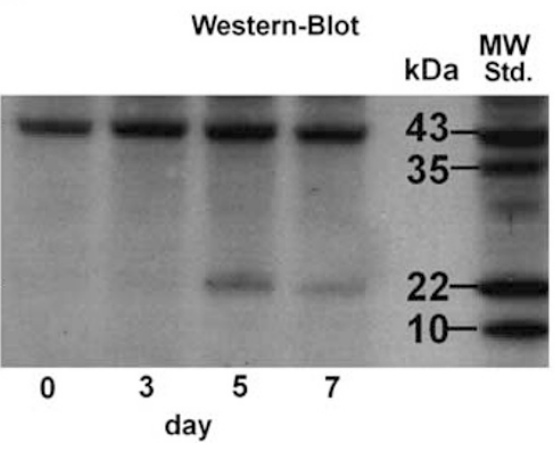

Figure 2 Transgelin is highly upregulated within mesangial cells during the repair phase in anti-Thy1 nephritis. Transgelin mRNA was found to be highly upregulated after anti-Thy1 nephritis induction using microarray technology (a). Glomerular upregulation of transgelin mRNA during anti-Thy1 nephritis was confirmed by real-time PCR (b) and northern-blot analysis (c). Representative transgelin protein expression was shown by western-blot analysis (d). In glomeruli from healthy rats, transgelin was below the detection level as assessed by immunohistochemistry (e). In contrast, migrating mesangial cells express transgelin 3 days after model induction (f, brown staining) and peak expression was reached on day 5 (g). ${ }^{*}$ Statistically significant changes between healthy (day 0 ) and injured glomeruli (days $1-7)$ tested by two-way ANOVA analysis $(P<0.05)$.

migration and the proliferating repopulating cell front that should be upregulated during anti-Thyl nephritis from day 2 up to day 5 . Hereby, either a continuously increasing gene expression up to day 5 (cluster 2, Table 1) or an increased expression with a peak on days $2 / 3$ (cluster 4 , Table 1 ) can be seen.

The gene with the most strikingly regulated expression pattern within cluster 2 was transgelin (SM actin 22-alpha). Therefore, it was studied in more detail as indicated below. Third, a specifically late occurring repair reaction (day 5 ) during anti-Thyl nephritis is represented by clusters 1 and 5, and may contain genes that are involved in the remodelling of the meanwhile well-established hypercellularity and matrix excess instead of the repair of the initial injury as mentioned for the other SOM clusters. Since this process is beyond the scope of this manuscript, these genes are neither mentioned nor studied in more detail.

\section{Transgelin as a Marker for the Repopulating Glomerular Cells}

Transgelin mRNA is detectable in normal kidney but only with a very low signal intensity (Figure 2a). Starting from day 1 , the expression is continuously upregulated $>8$-fold from day 1 to day 5 after antibody injection. This microarray data regarding transgelin mRNA are confirmed by real-time PCR (Figure 2b) and northern-blot analysis with an $1.0-\mathrm{kb}$ rat transgelin cDNA fragment (kindly provided by Dr C Shanahan) as a second independent method (Figure 2c). Whereas in normal kidney, transgelin mRNA is below the detection level of northern-blot analysis, the mRNA expression is significantly induced already on day 3 after injury and peaks on day 5. During the late phase of repair (day 7), the expression of transgelin already markedly ceases (Figures $2 \mathrm{~b}$ and c). This time course of transgelin expression was also confirmed by western-blot analysis (Figure $2 \mathrm{~d}$ ). To validate this result at the protein level and further characterize the transgelin-positive cell types during anti-Thyl nephritis, we performed immunostaining. Immunohistochemistry confirmed that transgelin was below the detection level in healthy glomeruli (Figure 2e), prominent in migrating MCs on day 3 (Figure 2f) and reaches its peak expression 5 days after nephritis induction (Figure $2 \mathrm{~g}$ ).

In Figure 3, representative pictures of transgelin and OX-7 single and double staining during anti-Thyl nephritis are depicted. In normal kidney, OX-7-positive but transgelinnegative MCs are the dominant cell type within the glomerulus (Figures $3 \mathrm{a}-\mathrm{c}$ ). Only renal vessels with a pericyte 

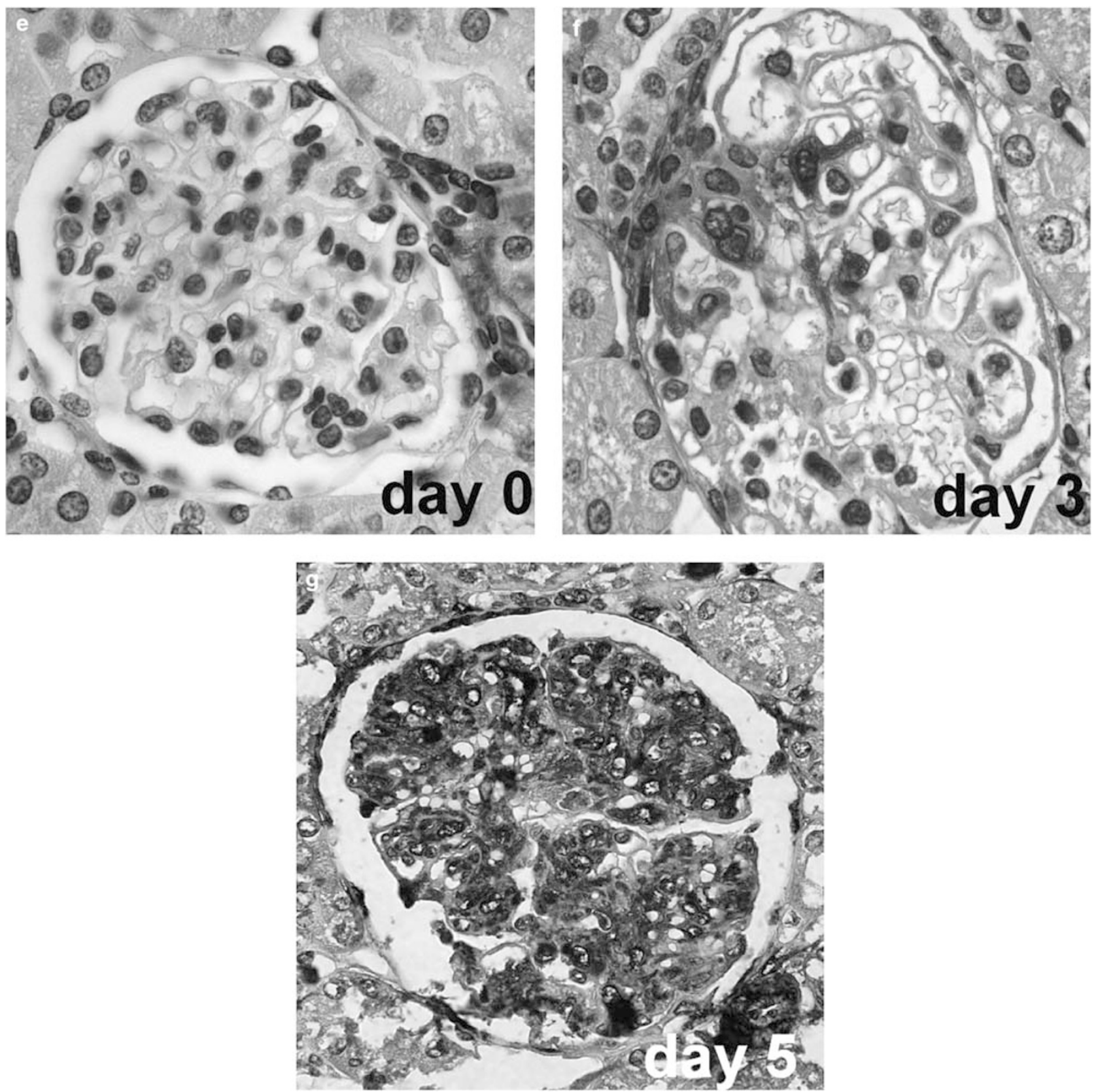

Figure 2 Conintued.

pattern are positive (green staining) for transgelin protein (Figure $3 \mathrm{~b}$ ), which are at the same time mostly negative for OX-7. Mesangiolysis on day 1 after disease induction is reflected by a marked decrease in OX-7-positive staining, ${ }^{1,2}$ while intraglomerular transgelin expression just starts to be upregulated (Figure 2). From day $2 / 3$ on, transgelin-positive cells can be detected within the glomerulus close to the extraglomerular mesangium and the hilar area and mainly colocalize with some but not all of the OX-7-positive MCs (Figure 3e, arrowhead). Reproducibly, transgelin-positive cells are predominantly located at the peripheral edge of the migrating OX-7-positive cell front (Figures $3 \mathrm{f}$ and i, arrow).
This process has almost maximally progressed on day 5 (Figures $3 \mathrm{~g}-\mathrm{i}$ ), where the glomerulus is almost completely repopulated by OX-7-positive cells (Figure $3 \mathrm{~g}$ ) and where transgelin-positive cells are still detectable at the edge of cell front towards glomerular periphery (Figure $3 \mathrm{~h}$ ). Finally, resting MCs seem to lose transgelin protein expression and become again negative for this marker (Figures 3f, i and l). Already on day 9 when repopulation is complete, intraglomerular transgelin staining has disappeared, but is still detectable at the hilar area in renal arteriolar pericytes (Figure 3k, arrowhead), while the hypercellular mesangium is still positive for the typical marker OX-7 (Figure 3j). 


\section{Transgelin Is Involved in MC Migration}

Since we detected transgelin primarily at the peripheral edge of the migrating MCs, we now investigated transgelins potential role in cell migration using a Boyden chamber assay. First, we examined whether transgelin expression is different in migrating $v s$ resting MCs. In contrast to MCs in the healthy glomerulus in vivo, cultured MCs are already in an activated state expressing also transgelin (Figure 4a). Three hours after seeding of MCs into the upper chamber of a transwell insert, we analysed transgelin expression of cells that passed the membrane (migrated cells) and cells remaining in the upper chamber using either western-blot analysis (Figure $4 \mathrm{~b}$ ) or flow cytometry (Figure 4c). Both methods clearly showed that transgelin expression was similar in migrating and non-migrating cells.

Next, we investigated whether a reduction of transgelin in MCs would influence their ability to migrate in the same Boyden chamber assay. Downregulation of transgelin expression (Figures $4 \mathrm{~d}-\mathrm{f}$ ) using specific siRNA reduced MC migration by $50 \%$ (Figure $4 \mathrm{~g}$ ).

\section{Transgelin Is Involved in MC Proliferation}

In anti-Thyl nephritis, MC repair is also characterized by cell proliferation. Most repopulating cells in anti-Thyl nephritis were double positive for transgelin and the proliferation marker PCNA (Figures 5a-c). Therefore, we investigated whether a reduction of transgelin in MCs would influence their ability to proliferate in vitro and monitored MC proliferation after treatment of cells with FCS. Transgelin knockdown via specific but not via control siRNA resulted in a $40 \%$ reduced proliferative activity, as measured by BrdUincorporation assay (Figure 5d). Next, we examined whether transgelin expression is regulated during cell-cycle progression using flow cytometry in cultured MC. Simultaneous staining for DNA content using propidium iodide for cell-cycle analysis (Figure 5e) and transgelin were performed.

Table 1 Differentially regulated genes classified by SOM clustering according to their gene expression profile

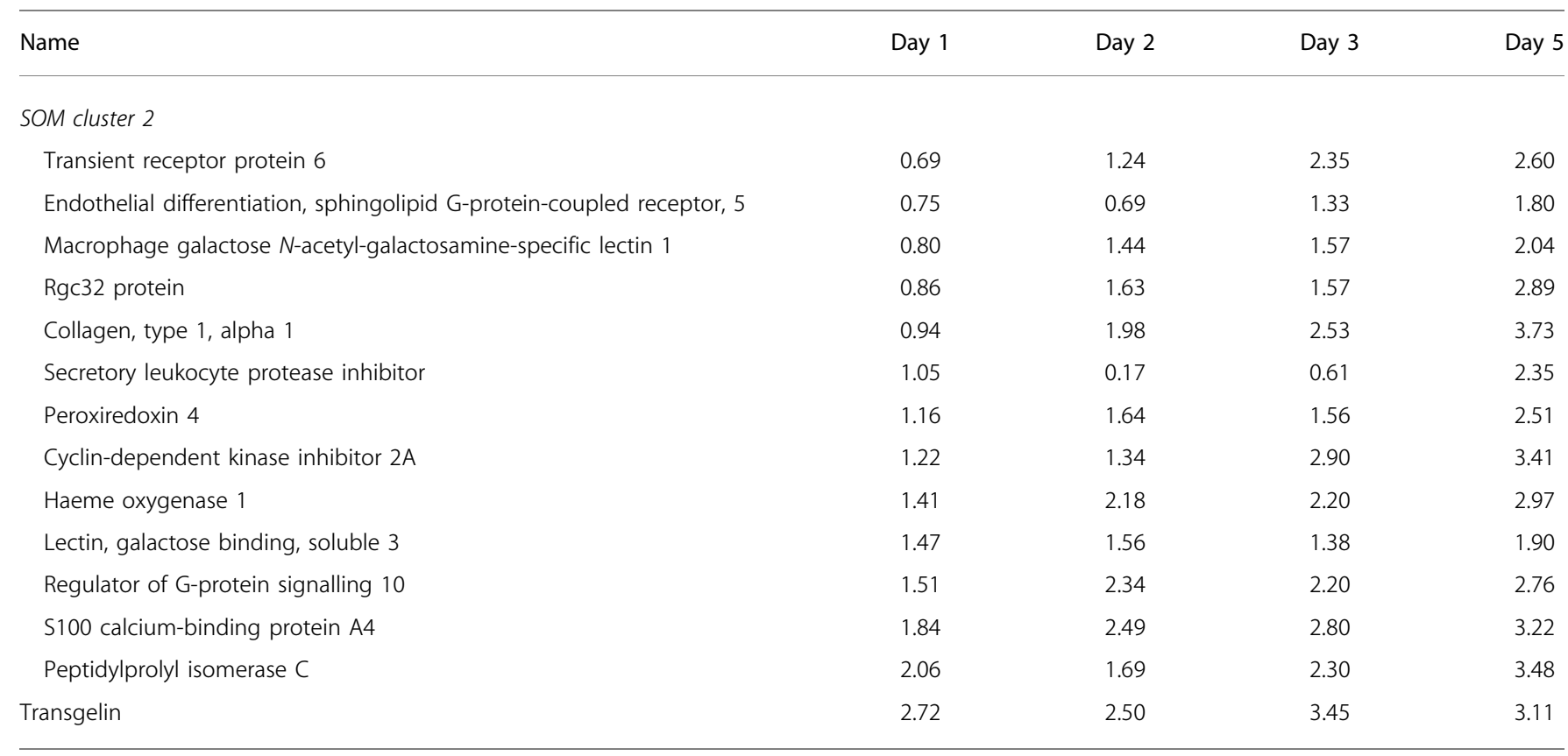

The values indicate the signal log ratio for each experimental time point compared with the untreated control. For a clearer summary, only those 15 genes that show the most prominent gene expression pattern within each cluster are indicated here.

Figure 3 Transgelin marks the leading edge of the repopulating glomerular cell front during anti-Thy 1 nephritis. In the left column, immunohistological staining of OX-7 (red colour; $\mathbf{a}, \mathbf{d}, \mathbf{g}, \mathbf{j}$ ), a marker for MCs is shown. The middle column illustrates the staining for transgelin (green colour; $\mathbf{b}$, e, $\mathbf{h}, \mathbf{k}$ ), whereas in the right column the overlay for OX-7 and transgelin is depicted (yellow; $\mathbf{c}, \mathbf{f}, \mathbf{i}, \mathbf{I})$. In the day 0 control (a-c), the tree-like mesangial pattern is shown by OX-7 staining (a) and transgelin is restricted to pericytes located at the vascular pole (b). During the ongoing glomerular repair process (days $2 / 3$ ), OX-7-positive cells reappear from the hilus (arrowheads) towards the periphery in a tree-like pattern (d), while transgelin-positive cells frequently spare a 'middle' area between the hilus and the leading edge of the repopulating cell front. Transgelin-positive cells are predominantly located at the leading edge of the migrating cell front towards glomerular periphery $(\mathbf{e}, \mathbf{f}$, arrow). This process is quite advanced on day 5 (g-i), when OX-7-positive cells have almost completely repopulated the glomerulus $(\mathbf{g})$ and transgelin-positive cells are most prominently detectable at the edge of cell front towards glomerular periphery $(\mathbf{h}, \mathbf{i}$, arrow). On day 9 after nephritis induction $(\mathbf{j}-\mathbf{l})$, hypercellularity of mesangial cells is still present (j), but transgelin positivity is completely lost $(\mathbf{k})$. 


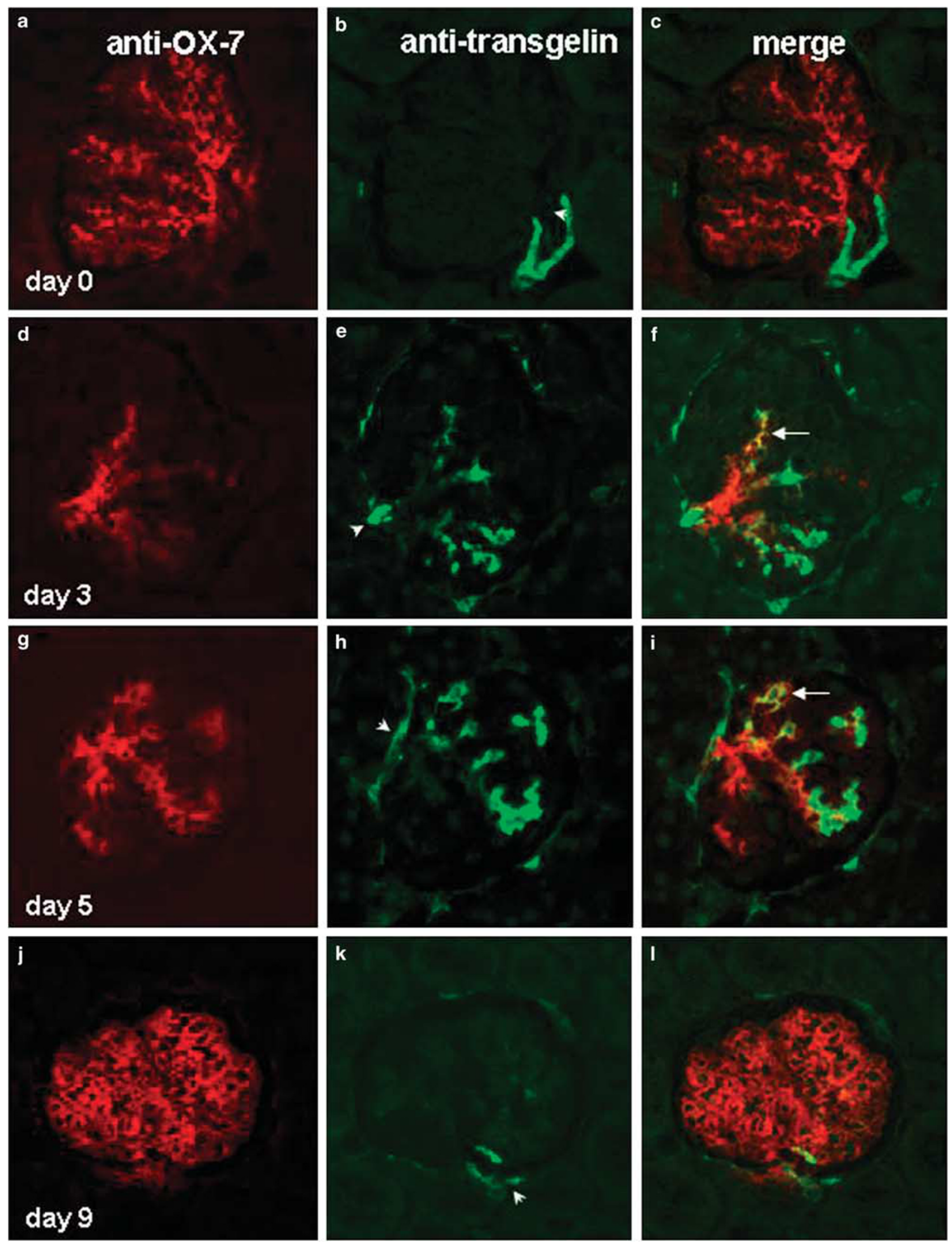


The number of cells showing a high intensity of the transgelin signal in G1, S and G2 phases increased continuously from 22 to $35 \%$ (Figures $5 \mathrm{f}-\mathrm{i}$ ), indicating that transgelin was slightly differentially expressed during cell-cycle progression.

\section{Mesangial Transgelin Expression during Glomerulogenesis}

Since mesangial proliferation, migration, and activation as indicated by positivity for alpha-SM actin are also important characteristics of MC in the developing kidney, we investigated transgelin expression during rat glomerulogenesis. During capillary loop stage, the first OX-7-positive cells could be detected within the developing glomeruli (Figure 6a). At this stage, the most pronounced transgelin staining was also detected within the MCs (Figure 6d). In further developed early glomeruli showing a simple morphology with OX-7-positive cells in the centre of the capillary convolute (Figure 6b) transgelin was prominent again within these MCs but also expressed in parietal epithelial cells and some podocytes (Figure 6e). In contrast, in early mature glomeruli, showing the typical tree-like OX-7 staining for MCs (Figure 6c), transgelin could be rarely detected (Figure 6f). Mesangial transgelin expression during glomerulogenesis was confirmed by confocal microscopy showing transgelin expression (Figure $6 \mathrm{~g}$, green fluorescence) and staining for the MC marker OX-7 (Figure 6h, red fluorescence) colocalizing in the centre of the developing glomerulus (Figure 6i).

\section{Transgelin was Expressed by MCs in Human IgA Nephritis}

Since anti-Thyl nephritis is a model for human IgA nephritis, we investigated transgelin expression in kidney biopsies from IgA nephritic patients compared with normal controls. In biopsies from healthy individuals only periglomerular, arteriolar renal pericytes show positive staining for transgelin similar to the pattern in normal rat glomeruli (Figure 7a). Transgelin could be detected in glomeruli from IgA nephritic patients showing early (Figures $7 \mathrm{~b}$ and c) as well as progressed chronic changes (Figures $7 \mathrm{~d}-\mathrm{f}$ ). Intensed staining for transgelin was detected also in Bowman's capsule and some podocytes, but prominent transgelin expression was also detected in MCs (Figure 7, arrows).

\section{DISCUSSION}

Administration of the monoclonal anti-Thy1 antibody OX-7 in rats induces reversible glomerulonephritis resembling many features of human mesangial proliferative disease. In contrast to human glomerulonephritis, which is frequently characterized by a slowly chronic progressive time course, the dramatically altered glomerular architecture during antiThyl nephritis is usually restored ad integrum very rapidly. ${ }^{19}$ Hereby, the transient hypercellularity and matrix excess is part of a complex and very successful glomerular healing reaction after severe injury that can be systematically studied. In this context, it is very important that certain specific modulations such as uninephrectomy, ${ }^{22}$ VEGF-blocking therapy, ${ }^{23}$ or everolimus (mTOR inhibitor) treatment ${ }^{24}$ during this very early repair reaction immediately after injury are sufficient to convert this reversible 'healing' model into a chronic progressive GN model similar to human disease. While this immediate response to injury reaction (due to the lack of symptoms) is always missed in human disease, this highly reproducible 'glomerular healing' model enables the investigation of important repair mechanisms.

The focus of this study was the molecular characterization of the process of glomerular repopulation, which occurs within several days after injury (after day 1 until day 5, ceasing after day 5) and predominantly results from directed migration and proliferation of surviving hilar or extraglomerular reserve cells towards the periphery of the glomerulus. ${ }^{1,2}$ While this fascinating mechanism appears to be important for a successful healing reaction, ${ }^{24}$ the phenotype of these repopulating cells as well as the signals triggering this process of repopulation after severe injury is hardly known. To define novel targets for regulation of glomerular repopulation, characterization of the repopulating glomerular cell front was a central goal of this study.

We identified transgelin as a new player/marker in repopulating MCs using an unsupervised gene clustering strategy (SOMs clustering). SOM cluster 2 appears to represent the characteristic gene expression pattern of this particular glomerular cell population. During repopulation, these cells express OX-7 indicating their mesangial character as well as alpha-SM actin and collagen I. All these marker proteins are not expressed in resting MCs of normal glomeruli ${ }^{1,2}$ as found in earlier studies and within cluster 2 of this study. An equivalent gene expression pattern (within cluster 2) also exhibits PTPRQ, which was already identified by our group via a PCR-based cDNA display approach. ${ }^{25}$ PTPRQ is specifically induced within the repopulating glomerular cells, ${ }^{25}$ confirming the validity of our approach and was recently identified as a phosphatidylinositol phosphatase. ${ }^{26}$

Among all genes of cluster 2, transgelin was most strikingly regulated during anti-Thyl nephritis. Hardly detectable in normal glomeruli, transgelin mRNA and protein were continuously upregulated up to day 5 after disease induction as shown by microarray and northern-blot analysis or immunostaining, respectively. In uninjured rats, transgelin-positive cells are predominantly restricted to the juxtaglomerular and arteriolar pericytes, which are mostly OX-7 negative. After glomerular injury, transgelin-positive cells are predominantly located at the peripheral edge of the migrating OX-7-positive cell front, suggesting a role in migration/proliferation or differentiation of these repopulating cells. Interestingly, during repopulation a transgelin negative, OX-7-positive cell population could be reproducibly identified between the hilus and the double (transgelin/OX-7)-positive cell front at the leading edge. Whether this OX-7 positive, transgelin-negative cell 
a

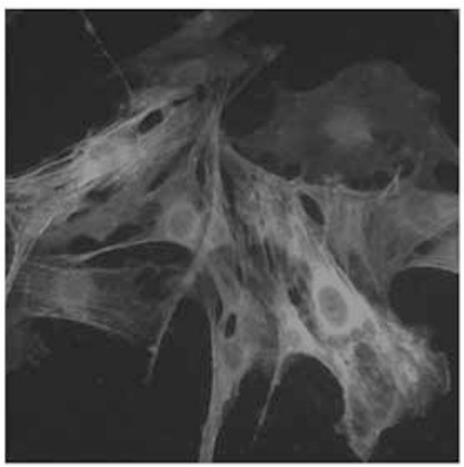

C

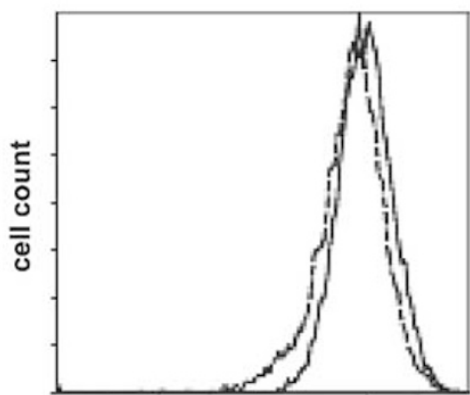

FL1 [anti-transgelin]

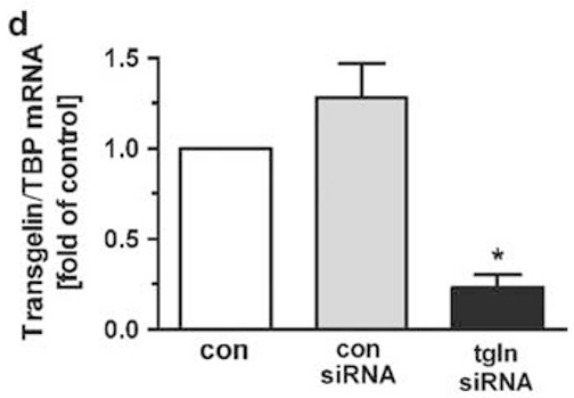

f

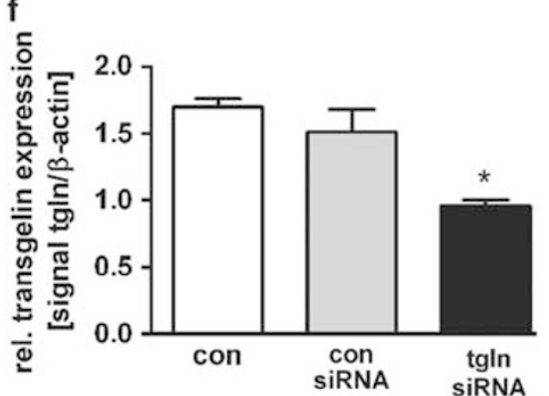

b
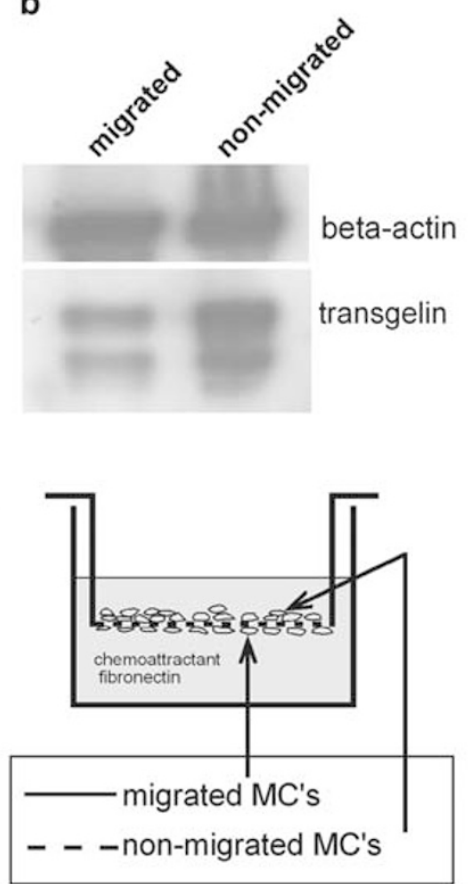

e

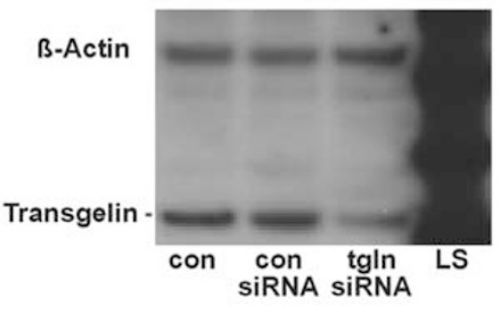

g

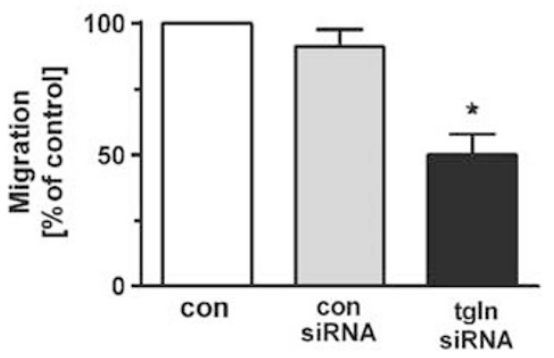

Figure 4 Transgelin promotes mesangial cell migration. In contrast to mesangial cells in healthy glomeruli, isolated rat mesangial cells express transgelin as shown by immunofluorescence staining (a, green fluorescence). Analysis of migrated vs non-migrated mesangial cells by western-blot analysis (b) or flow cytometry (c) showed no significant different transgelin expression in both populations. Using siRNA technology, transgelin expression was reduced in isolated mesangial cells as assessed at the mRNA level by real-time PCR (d) and at the protein level by western-blot analysis (e) as analysed by densitometry (f). Mesangial cell migration was reduced by $50 \%$ in mesangial cells treated with transgelin-specific siRNA but not in cells treated with a control siRNA (g). * Statistically significant changes between controls and siRNA treated MC tested by two-way ANOVA analysis $(P<0.05)$.

population has already settled down and is back to a 'resting/ contractile' phenotype is speculative but consistent with the known function of transgelin so far and our data within this manuscript. This remarkable expression pattern is also in agreement with both possibilities that after injury juxtaglomerular MC-like reserve cells acquire an activated phenotype during repopulation or that juxtaglomerular precursor cells divide and migrate into the glomerulus to differentiate into an $\mathrm{MC}$ while losing certain phenotypic characteristics. 

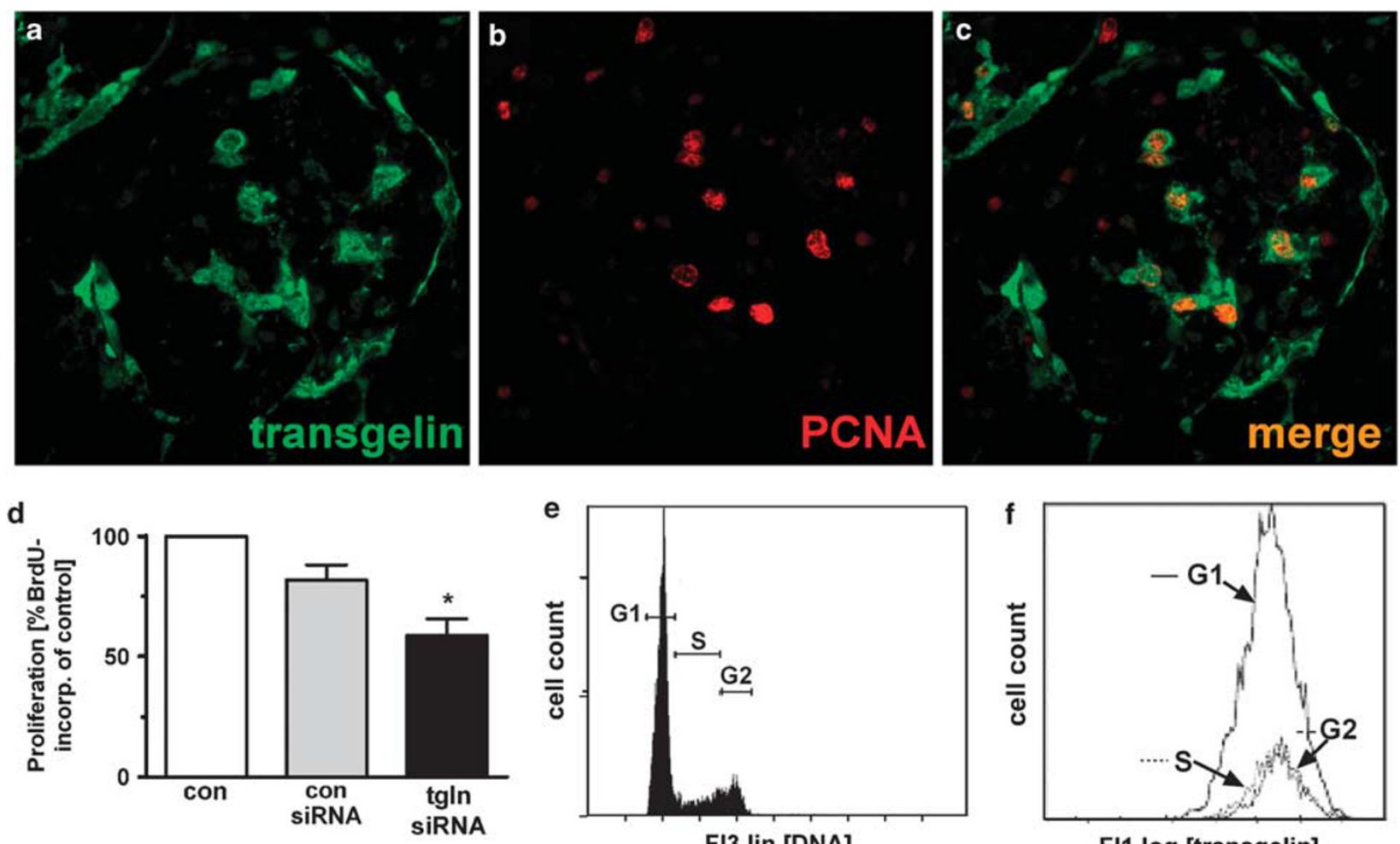

$\mathrm{F} I 3 \operatorname{lin}[\mathrm{DNA}]$

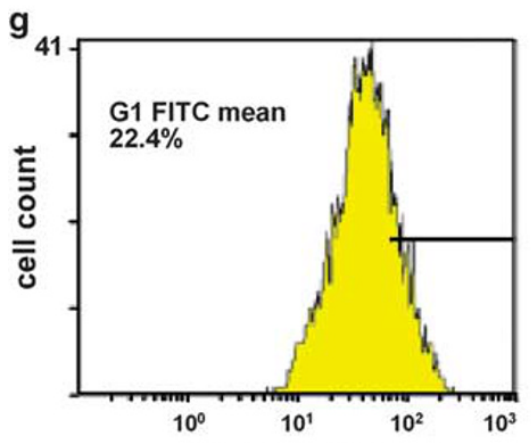

h
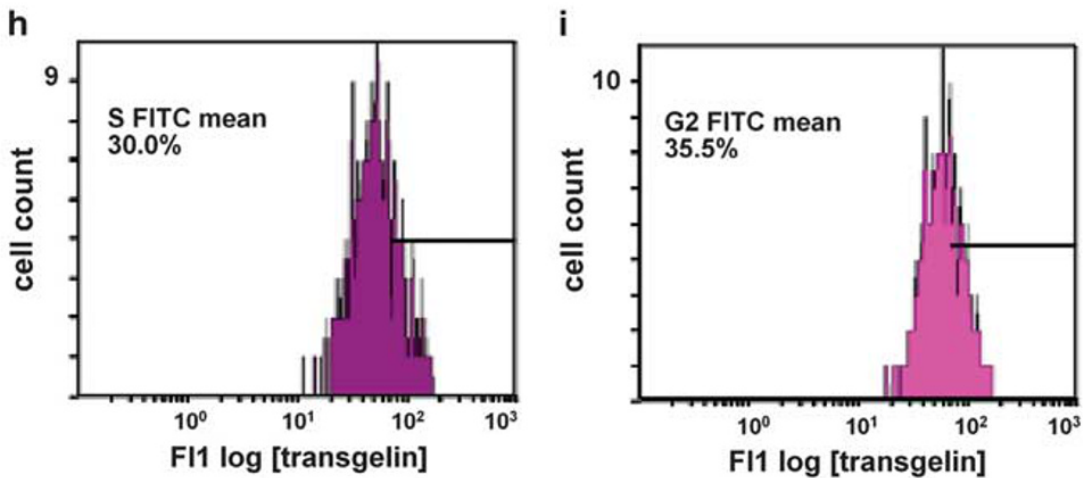

Figure 5 Transgelin promotes mesangial cell proliferation. Double immunostaining in kidney biopsies from anti-Thy 1 nephritic rats on day 5 shows that transgelin (a, green fluorescence) and the proliferation marker PCNA (b, red fluorescence) colocalizes within the glomeruli (c, orange merged fluorescence). Treatment of isolated rat mesangial cells with transgelin-specific siRNA resulted in a $40 \%$ inhibition of BrdU incorporation, which was not seen using control siRNA (d). Mesangial cells were stained with propidium iodide for monitoring of cell cycle by measurement of flow cytometric DNA content (e). In parallel, mesangial cells were stained for transgelin for cell-cycle phase-dependent analysis (f). Cells were gated for cell-cycle phases G1 (g), S phase (h), and G2 phase (i). The percentage of cells expressing high levels of transgelin was determined during the G1, S, and G2 phases (g-i). * Statistically significant changes between controls and siRNA treated MC tested by two-way ANOVA analysis $(P<0.05)$.

Transgelin is an actin-binding $22-\mathrm{kDa}$ protein abundantly and exclusively expressed in smooth muscle cells of adult animals. ${ }^{27}$ While the analysis of transgelin-deficient mice demonstrated no developmental or basal homeostatic functions of transgelin in smooth muscle cells ${ }^{27}$ further studies implicate a critical role under pathophysiological settings. ${ }^{28}$ In hypercholesterolemic ApoE-deficient mice, the lack of transgelin resulted in increased atherosclerotic lesion area and a higher proportion of proliferating smooth muscle cell-derived plaque cells. ${ }^{28}$ This suggests that transgelin inhibits phenotypic modulation of smooth muscle cells from a contractile to a synthetic/proliferative cell type. Studies in transgelin-deficient smooth muscle cells in vitro demonstrate a role of transgelin in contractility, possibly by promoting actin filament stability. ${ }^{29}$ In addition, transgelin was markedly upregulated by TGF- $\beta$ induced transdifferentiation of prostate fibroblasts into myofibroblasts. ${ }^{30}$

Cellular migration and proliferation of MCs, the glomerular pericytes, are the two key features that are important for repopulation and regeneration of the glomerulus after 

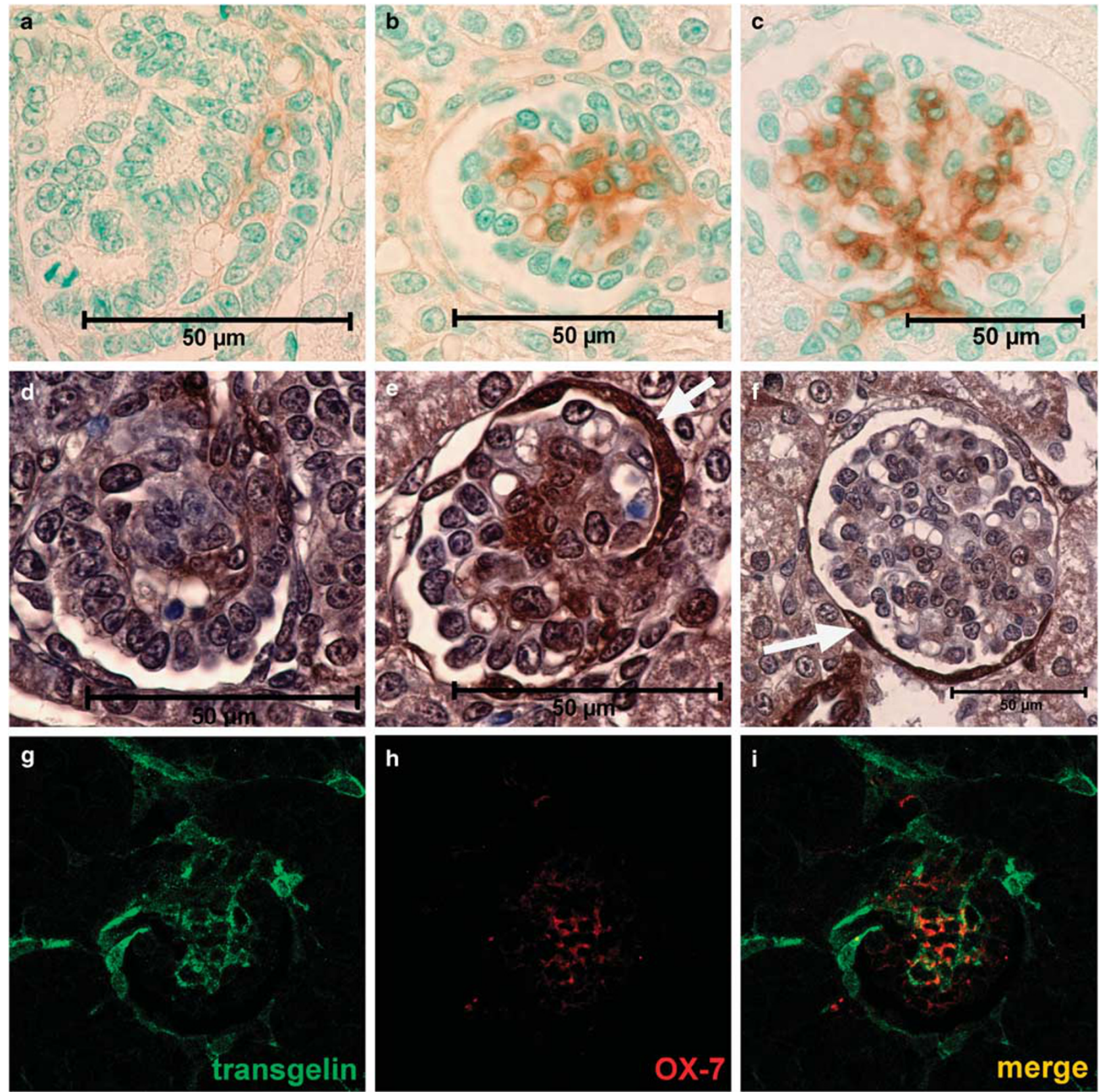

Figure 6 Transgelin is expressed in mesangial cells during glomerulogenesis. Kidneys from 6-day-old rats are stained for the mesangial marker OX-7 (a-c) and transgelin (d-f) using immunohistochemistry. During capillary loop stage, OX-7-positive mesangial cells are detectable in the developing glomeruli (a). Transgelin can be detected at a similar localization (d). Later stages of developing glomeruli express OX-7 (b) as well as transgelin (e) in the centre of the glomerulus. In addition, parietal epithelial cells (e, white arrow) and lightly some podocytes also express transgelin. Early mature glomeruli show the typical tree-like OX-7 staining for mesangial cells (c). At this stage, glomerular transgelin staining still decreases (f) whereas transgelin expression of parietal epithelial cells remains prominent (f, white arrow). Developing glomeruli were also analysed by immunofluorescence staining for transgelin (g, green fluorescence staining) together with OX-7 (h, red fluorescence staining). Colocalization of both markers (i) is shown using confocal microscopy.

mesangiolysis in the anti-Thy 1 model. Despite incomplete downregulation of transgelin by siRNA technology, our studies in vitro clearly show a marked promigratory function of transgelin in isolated MCs. A role of transgelin in cell migration was also found in different non-renal cell types. ${ }^{31,32}$ TGF- $\beta$ induced cell migration of human alveolar epithelial cells ${ }^{32}$ and the migration and invasiveness of hepatocellular cancer stem cells ${ }^{31}$ could be significantly inhibited by knockdown of transgelin, suggesting a role in cytoskeletal organization facilitating movement of cells. In our study, transgelin was also involved in MC proliferation, since transgelin knockdown resulted in a significantly reduced proliferative activity. While transgelin shows pro-proliferative effects in human epithelial cells, ${ }^{32}$ proliferation 

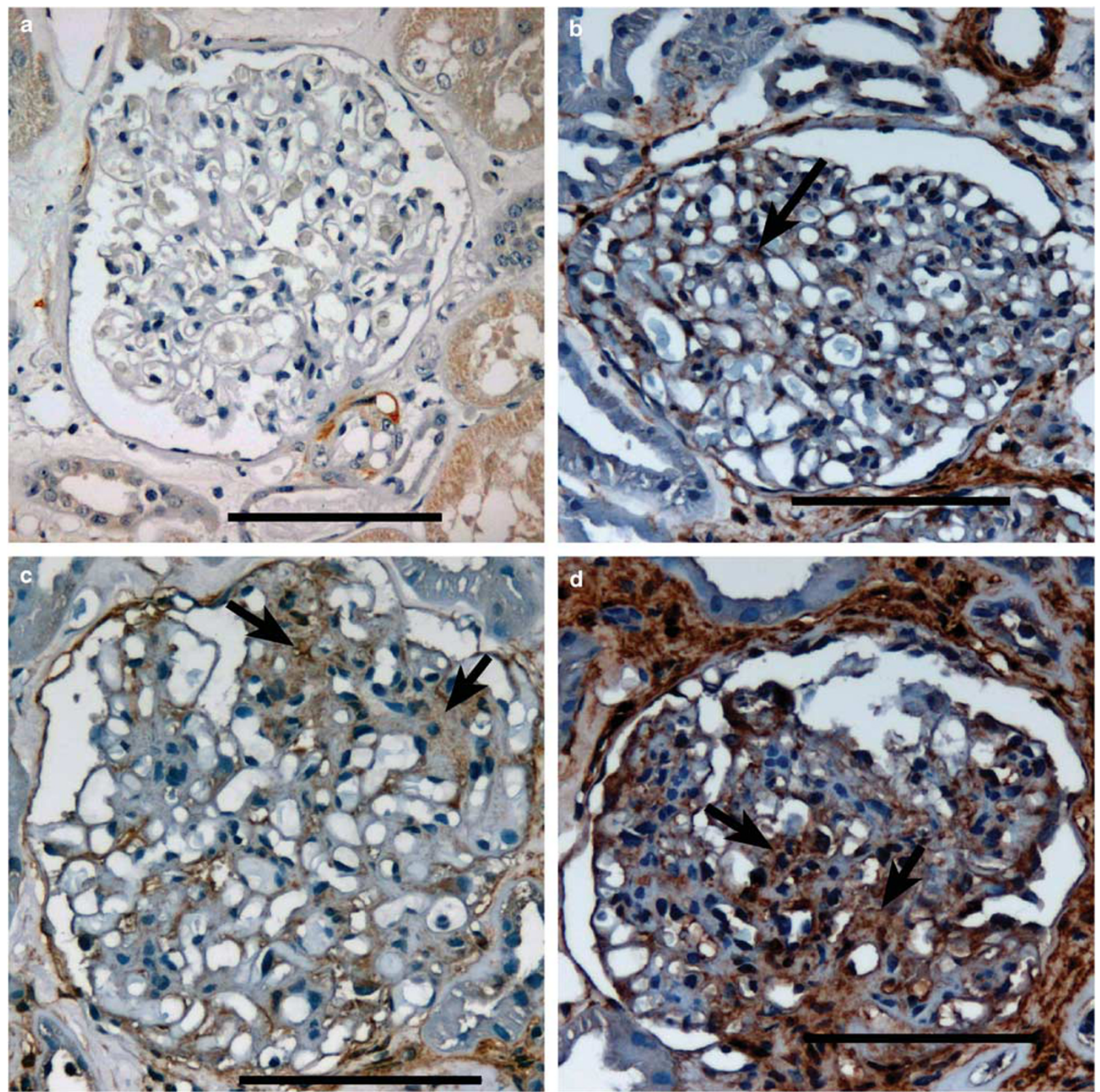

Figure 7 Transgelin expression in biopsies from patients with IgA nephropathy. In healthy glomeruli, transgelin can be detected only in a typical pericyte pattern at the glomerular arteriole using immunohistochemistry (a). In biopsies from patients with IgA nephropathy, transgelin is expressed in glomeruli showing mild pathological changes $(\mathbf{b}, \mathbf{c})$ as well as in glomeruli with advanced disease progression (d-f). Transgelin-positive cells in locations typical for mesangial cells are marked with arrows. Magnification in (a-f) is indicated by a black bar representing a distance of $100 \mu \mathrm{m}$.

of prostate tumour cells was enhanced by downregulation of transgelin, ${ }^{33}$ indicating cell-specific transgelin effects on proliferation.

MC migration as well as proliferation is also important during glomerulogenesis. Interestingly, we could detect transgelin predominantly in $\mathrm{MC}$ as well as in parietal epithelial cells and much weaker in some podocytes during early glomerulogenesis in rats. In contrast, transgelin in mice was only detected in parietal epithelial cells and podocytes. ${ }^{34}$
Transgelin was found to be upregulated in different renal injury models like anti-GBM nephritis, ${ }^{34-36}$ passive Heymann nephritis, ${ }^{34,37}$ the remnant kidney model and puromycin aminoglycoside nephritis ${ }^{34,38}$ but also in human glomerulonephritis. ${ }^{39}$ While timely and spatially restricted upregulation of transgelin is likely to be supportive for repair processes such as MC proliferation and migration after acute reversible mesangiolysis, this approach is not necessarily a therapeutic goal in the situation of chronic, progressive 

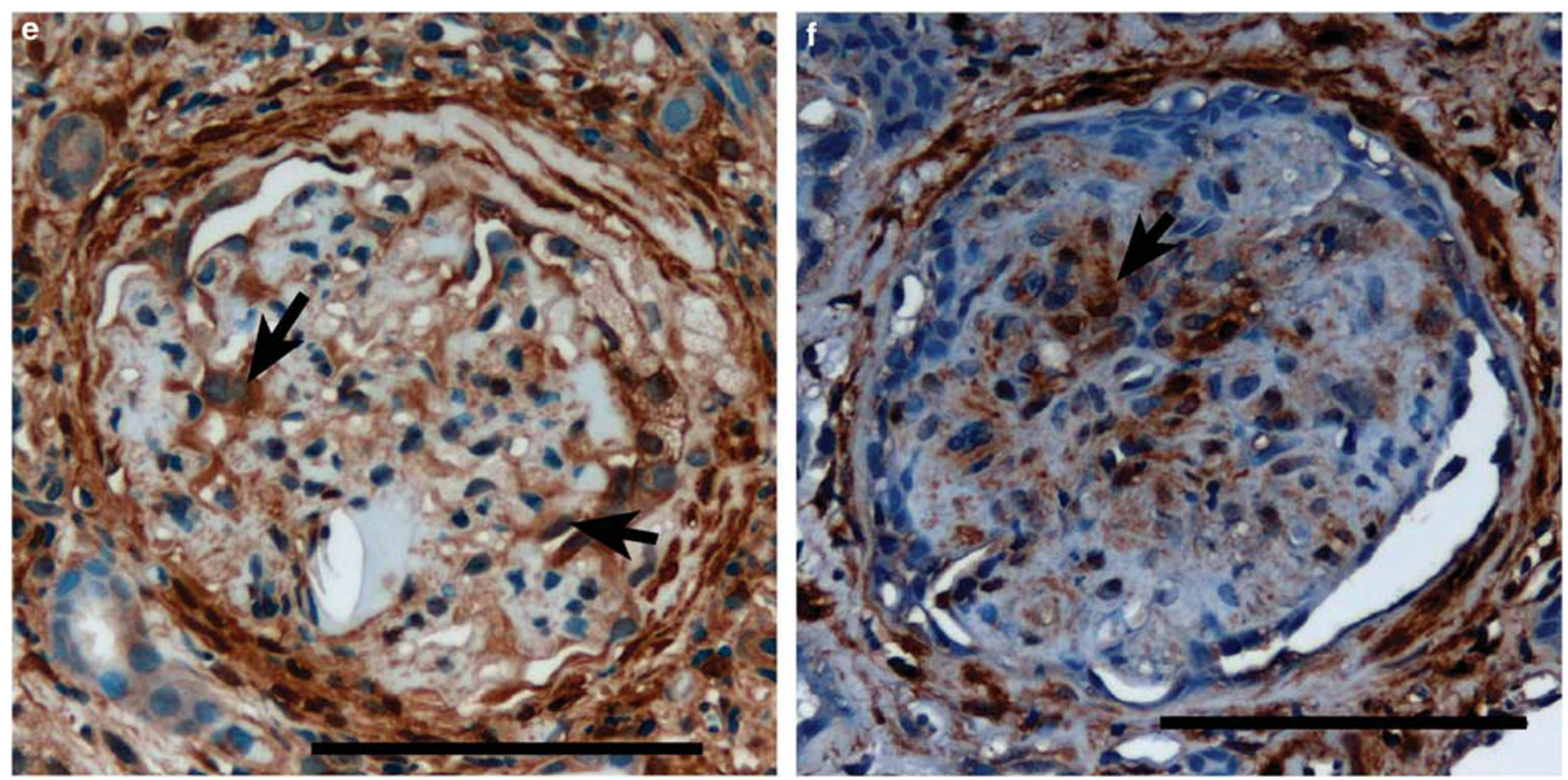

Figure 7 Conintued.

glomerulonephritis such as IgA nephropathy. Hereby, increased transgelin may be an indicator for a dysregulatory or compensatory condition, in which suppression of transgelin may be more beneficial. For example, Marshall et al $l^{34}$ demonstrated that GBM-nephritic mice lacking the transgelin gene developed less severe histopathology compared with nephritic wild-type mice. In addition, transgelin expression was correlated with histopathologic parameters in biopsies of glomerulonephritis patients showing a positive correlation between the severity of transgelin expression with the degree of mesangial proliferation and glomerulosclerosis. ${ }^{39}$ However, mild transgelin expression was related to a favourable clinical course. Especially for patients with membranous nephropathy the severity of glomerular transgelin correlated with a higher remission rate of proteinuria. ${ }^{39}$ In contrast, higher interstitial transgelin expression correlated with an unfavourable clinical course, ${ }^{39}$ indicating that transgelin expression is not per se beneficial and induced effects are may be cell type specific.

Transgelin is only one component of several orchestrating renal repair. As shown in the table, there are more candidates or players that may be important during this process. We identified further transcriptionally regulated genes, but several interesting molecules involved in cell migration/motility like $\mathrm{Cdc} 42$, focal adhesion kinase, and raf are presumably activated via phosphorylation events and translocation within the cell rather than upregulation of their gene expression. In the present study, their gene products are detectable but not regulated over the time course of disease (data not shown). Nevertheless, the striking functional relevance of an only partial reduction of a single factor such as transgelin in our in-vitro migration and proliferation assays underlines the importance of this particular factor identified via microarray analysis.

In summary, we identified transgelin as a new marker for repopulating MCs during the early repair phase of anti-Thy1 nephritis promoting cellular migration and proliferation.

Supplementary Information accompanies the paper on the Laboratory Investigation website (http://www.laboratoryinvestigation.org)

\section{ACKNOWLEDGEMENTS}

This study was supported by the Interdisciplinary Center for Clinical Research (IZKF) at the University Hospital of the University of ErlangenNuremberg (TP A12) with funding by the Federal Ministry of Education and Research (Fund No. 01 KS 0002). We thank Dr C Shanahan (University of Cambridge, Cambridge, UK) for the support of the CDNA fragment for transgelin. We gratefully appreciate the skilled technical help of Andrea Stief, Tanja Christ, Ulrike Goller, Andrea Lüdke, Christina Grigo and Susanne Weber.

\section{DISCLOSURE/CONFLICT OF INTEREST}

The authors declare no conflict of interest.

1. Hugo C, Shankland SJ, Bowen-Pope DF, et al. Extraglomerular origin of the mesangial cell after injury. A new role of the juxtaglomerular apparatus. J Clin Invest 1997;100:786-794.

2. Hugo $C$, Hugo $C$, Pichler $R$, et al. The cytoskeletal linking proteins, moesin and radixin, are upregulated by platelet-derived growth factor, but not basic fibroblast growth factor in experimental mesangial proliferative glomerulonephritis. J Clin Invest 1996;97:2499-2508.

3. Butte A. The use and analysis of microarray data. Nat Rev Drug Discov 2002;1:951-960.

4. Alcorta DA, Prakash K, Waga I, et al. Future molecular approaches to the diagnosis and treatment of glomerular disease. Semin Nephrol 2000;20:20-31.

5. Peterson KS, Huang JF, Zhu J, et al. Characterization of heterogeneity in the molecular pathogenesis of lupus nephritis from transcriptional profiles of laser-captured glomeruli. J Clin Invest 2004;113:1722-1733. 
6. Schwab K, Witte DP, Aronow BJ, et al. Microarray analysis of focal segmental glomerulosclerosis. Am J Nephrol 2004;24:438-447.

7. Alcorta $D$, Preston $G$, Munger W, et al. Microarray studies of gene expression in circulating leukocytes in kidney diseases. Exp Nephrol 2002:10:139-149.

8. Wada J, Zhang H, Tsuchiyama $Y$, et al. Gene expression profile in streptozotocin-induced diabetic mice kidneys undergoing glomerulosclerosis. Kidney Int 2001;59:1363-1373.

9. Ohse T, Ota T, Kieran N, et al. Modulation of interferon-induced genes by lipoxin analogue in anti-glomerular basement membrane nephritis. J Am Soc Nephrol 2004;15:919-927.

10. Izumi $Y$, Izumiya $Y$, Shiota $M$, et al. Gene expression profile in experimental mesangial proliferative glomerulonephritis. J Pharmacol Sci 2004;96:91-94.

11. Ito T, Suzuki A, Imai E, et al. Bone marrow is a reservoir of repopulating mesangial cells during glomerular remodeling. J Am Soc Nephrol 2001;12:2625-2635.

12. Daniel C, Renders L, Amann K et al. Mechanisms of everolimusinduced glomerulosclerosis after glomerular injury in the rat. Am J Transplant 2005;5:2849-2861.

13. Rost S, Daniel C, Schulze-Lohoff E, et al. P2 receptor antagonist PPADS inhibits mesangial cell proliferation in experimental mesangial proliferative glomerulonephritis. Kidney Int 2002;62:1659-1671.

14. Durig J, Nuckel H, Huttmann A, et al. Expression of ribosomal and translation-associated genes is correlated with a favorable clinical course in chronic lymphocytic leukemia. Blood 2003;101:2748-2755.

15. Shanahan CM, Weissberg PL, Metcalfe JC. Isolation of gene markers of differentiated and proliferating vascular smooth muscle cells. Circ Res 1993:73:193-204.

16. Johnson $\mathrm{RJ}$, lida $\mathrm{H}$, Alpers $\mathrm{CE}$, et al. Expression of smooth muscle cell phenotype by rat mesangial cells in immune complex nephritis. Alphasmooth muscle actin is a marker of mesangial cell proliferation. J Clin Invest 1991:87:847-858.

17. Wittmann S, Daniel C, Stief A, et al. Long-term treatment of sirolimus but not cyclosporine ameliorates diabetic nephropathy in the rat Transplantation 2009;87:1290-1299.

18. Daniel $\mathrm{C}$, Albrecht $\mathrm{H}$, Ludke $A$, et al. Nestin expression in repopulating mesangial cells promotes their proliferation. Lab Invest 2008;88: 387-397.

19. Jefferson JA, Johnson RJ. Experimental mesangial proliferative glomerulonephritis (the anti-Thy-1.1 model). J Nephrol 1999;12:297-307.

20. Tamayo P, Slonim D, Mesirov J, et al. Interpreting patterns of gene expression with self-organizing maps: Methods and application to hematopoietic differentiation. PNAS 1999;96:2907-2912.

21. Toronen $\mathrm{P}$, Kolehmainen $\mathrm{M}$, Wong $\mathrm{G}$, et al. Analysis of gene expression data using self-organizing maps. FEBS Lett 1999;451:142-146.

22. Cheng $\mathrm{QL}$, Orikasa $\mathrm{M}$, Morioka $\mathrm{T}$, et al. Progressive renal lesions induced by administration of monoclonal antibody 1-22-3 to unilaterally nephrectomized rats. Clin Exp Immunol 1995;102:181-185.

23. Ostendorf T, Kunter U, Eitner F, et al. $\operatorname{VEGF(165)~mediates~glomerular~}$ endothelial repair. J Clin Invest 1999;104:913-923.
24. Daniel C, Pippin J, Shankland SJ, et al. The rapamycin derivative RAD inhibits mesangial cell migration through the CDK-inhibitor p27KIP1. Lab Invest 2004;84:588-596.

25. Wright $M B$, Hugo $C$, Seifert $R$, et al. Proliferating and migrating mesangial cells responding to injury express a novel receptor proteintyrosine phosphatase in experimental mesangial proliferative glomerulonephritis. J Biol Chem 1998;273:23929-23937.

26. Oganesian A, Poot M, Daum G, et al. Protein tyrosine phosphatase RQ is a phosphatidylinositol phosphatase that can regulate cell survival and proliferation. PNAS 2003;100:7563-7568.

27. Zhang JC, Kim S, Helmke BP, et al. Analysis of SM22alpha-deficient mice reveals unanticipated insights into smooth muscle cell differentiation and function. Mol Cell Biol 2001;21:1336-1344.

28. Feil S, Hofmann F, Feil R. SM22alpha modulates vascular smooth muscle cell phenotype during atherogenesis. Circ Res 2004;94: 863-865.

29. Zeidan A, Sward K, Nordstrom I, et al. Ablation of SM22alpha decreases contractility and actin contents of mouse vascular smooth muscle. FEBS Lett 2004;562:141-146.

30. Untergasser G, Gander R, Lilg $C$, et al. Profiling molecular targets of TGF-beta1 in prostate fibroblast-to-myofibroblast transdifferentiation. Mech Ageing Dev 2005;126:59-69.

31. Lee EK, Han GY, Park HW, et al. Transgelin promotes migration and invasion of cancer stem cells. J Proteome Res 2010;9:5108-5117.

32. Yu H, Konigshoff M, Jayachandran A, et al. Transgelin is a direct target of TGF-beta/Smad3-dependent epithelial cell migration in lung fibrosis. FASEB J 2008:22:1778-1789.

33. Yang $\mathrm{Z}$, Chang $\mathrm{YJ}$, Miyamoto $\mathrm{H}$, et al. Transgelin functions as a suppressor via inhibition of ARA54-enhanced androgen receptor transactivation and prostate cancer cell growth. Mol Endocrinol 2007;21:343-358.

34. Marshall CB, Krofft RD, Blonski MJ, et al. Role of smooth muscle protein SM22alpha in glomerular epithelial cell injury. Am J Physiol Renal Physiol 2011;300:F1026-F1042.

35. Ogawa A, Sakatsume $M$, Wang $X$, et al. SM22alpha: the novel phenotype marker of injured glomerular epithelial cells in antiglomerular basement membrane nephritis. Nephron Exp Nephrol 2007; 106:e77-e87.

36. Sakamaki $Y$, Sakatsume $M$, Wang $X$, et al. Injured kidney cells express SM22alpha (transgelin): unique features distinct from alpha-smooth muscle actin (alphaSMA). Nephrology (Carlton) 2011; 16:211-218.

37. Hauser PV, Perco P, Muhlberger I, et al. Microarray and bioinformatics analysis of gene expression in experimental membranous nephropathy. Nephron Exp Nephrol 2009;112:e43-e58.

38. Inomata S, Sakatsume M, Sakamaki Y, et al. Expression of SM22alpha (transgelin) in glomerular and interstitial renal injury. Nephron Exp Nephrol 2011;117:e104-e113.

39. Gerolymos $M$, Karagianni $F$, Papasotiriou $M$, et al. Expression of transgelin in human glomerulonephritis of various etiology. Nephron Clin Pract 2011;119:c74-c82. 